\title{
Effects of blend of canola oil and palm oil on nutrient intake and digestibility, growth performance, rumen fermentation and fatty acids in goats
}

\begin{abstract}
The study examined the effects of blend of $80 \%$ canola oil and $20 \%$ palm oil (BCPO) on nutrient intake and digestibility, growth performance, rumen fermentation and fatty acids (FA) in goats. Twenty-four Boer bucks were randomly assigned to diets containing 0,4 and $8 \%$ BCPO on a dry matter basis, fed for 100 ldlays and slaughtered. Diet did not affect feed efficiency, growth performance, intake and digestibility of all nutrients except ether extract. Intakes and digestibilities of ether extract, unsaturated fatty acids (FA) and total FA were higher (P\&IOL) while digestibility of C18:0 was lower (P\&IOL) in oil-fed goats than the control goats. Total volatile FA, acetate, butyrate, acetate/propionate ratio and methane

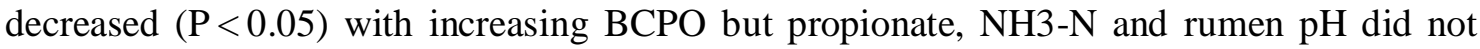
differ between diets. Ruminal concentration of C18:0, n-3 FA and total FA increased (P\&\&l.05) while C12:0, C14:0, C15:0 and n-6 FA decreased with increasing BCPO. Analysis of the FA composition of Triceps brachii muscle showed that concentrations of C16:0, C14:0 and C18:2n-6 were lower (P度l.05) while C18:1n-9, C18:3n-3 and C20:5n-3 were higher in oil-fed goats compared with control goats. Dietary BCPO altered muscle lipids without having detrimental effects on nutrient intake and digestibility and growth performance in goats.
\end{abstract}

Keyword: Digestibility; Fatty acid; Goat; Rumen fermentation; Triceps brachii 